\title{
Homopolar Permanent-Magnet-Biased Actuators and Their Application in Rotational Active Magnetic Bearing Systems
}

\author{
Alexei Filatov*, Larry Hawkins and Patrick McMullen \\ Calnetix Technologies LLC, 16323 Shoemaker Av., Cerritos, CA 90703, USA; lhawkins@calnetix.com (L.H.); \\ pmcmullen@calnetix.com (P.M.) \\ * Correspondence: afilatov@calnetix.com; Tel.: +1-562-293-1667 \\ Academic Editor: Eric H. Maslen \\ Received: 4 November 2016; Accepted: 14 December 2016; Published: 16 December 2016
}

\begin{abstract}
Active Magnetic Bearings (AMBs) are already widely used in rotating machinery and continue to gain popularity due to the ever-present push to higher rotational speeds and decreasing prices of associated electronic components. They offer several advantages over conventional mechanical bearings including non-contact rotor support (thus eliminating mechanical wear and the need for lubricants), ability to tune bearing parameters through software for optimum machine performance, remote monitoring and health diagnostic, etc. In some applications, such as in a vacuum or in aggressive environments, they are often the only viable solution. An electromagnetic actuator, along with a position sensor and control electronics, is a key component of AMBs. While there is a variety of actuator designs described in the literature, most of the AMBs built commercially use heteropolar radial electrical actuators in combination with a dedicated electrically-biased axial actuators. On the contrary, since its inception in 1998, Calnetix Technologies mainly uses homopolar permanent magnet (PM)-biased radial actuators along with a homopolar PM-biased combination radial/axial actuators. In this paper, we provide an overview of the research we have done over the last 15 years in this area focusing on the advantages and disadvantages of this approach and explaining why we have made certain design choices.
\end{abstract}

Keywords: electromagnetic actuators; Active Magnetic Bearings; permanent magnets; rotating machinery; rotordynamics

\section{Introduction: Electromagnetic Actuators as Part of Active Magnetic Bearing Systems}

The operational principle of the Active Magnetic Bearings (AMB) is rather simple [1]:

- An electromagnetic actuator is configured to exert forces on a supported ferromagnetic object.

- The object position in space is monitored by a position sensor.

- The position sensor provides information about the object position to a control system.

- The control system adjusts the current in the actuator in response to changes in the position and an application specific control input.

- Force is applied by the actuator on the object to maintain the object in the desired position in space without coming into mechanical contact.

This operational principle is schematically illustrated in Figure 1 using a simple Horse-Shoe electromagnet as an example of an electromagnetic actuator. 


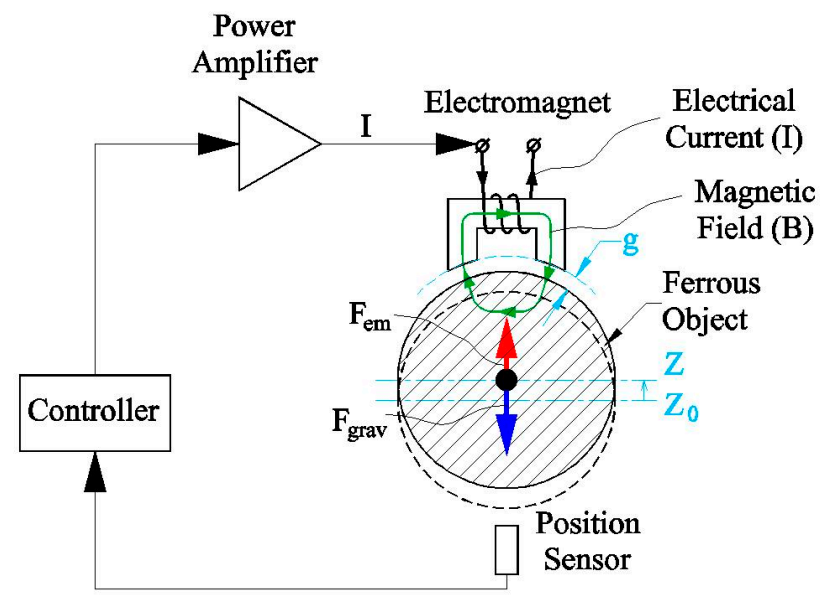

Figure 1. A schematic illustration of the AMB operating principle. $F_{e m}$ : electromagnetic force; $F_{\text {grav }}$ : gravity force; $g$ : gap between the electromagnet and the object; $Z$ : object position; $Z_{0}$ : targeted levitation position.

The pulling force exerted by the electromagnet on the object is always attractive and depends on two parameters:

(a). Current $I$ in the electromagnet.

(b). Gap between the object and the electromagnet $g$, also represented by the object position $Z$.

If the current $I$ does not change, the force $F_{e m}$ pulling the object towards the electromagnet will get stronger when the rotor gets closer to the electromagnet. Because of this, and because the gravity force $F_{\text {grav }}$ effectively does not change with the rotor position, if the rotor is brought close enough to the electromagnet for the electromagnetic force $F_{e m}$ to overcome the gravity force $F_{\text {grav }}$, the rotor will jump to the electromagnet and stick to it. Alternatively, if the rotor is placed too far from the electromagnet where the gravity force is larger than electromagnetic force, the rotor will fall on the floor.

This behavior is just the opposite to what would happen if the rotor was suspended on a spring as shown in Figure 2-the force with which the spring would pull the rotor up would get weaker when the rotor moves up and stronger when the rotor moves down. The spring suspension shown in Figure 2 is said to have a positive stiffness (defined as a rate at which spring force changes with the rotor displacement taken with a negative sign) whereas the electromagnet suspension shown in Figure 1 has a negative stiffness. We know that an object can be suspended on a spring as shown in Figure 2, but it is not possible to suspend an object using the electromagnet shown in Figure 1 energized with a constant current without it coming into full contact with the electromagnet.

In order to achieve stable levitation of a rotor in an $A M B$, the current in the electromagnet is continuously adjusted to keep the rotor in the desired position $Z_{0}$. The position $Z$ is constantly measured using a position sensor, shown in Figure 1, which passes information to an electronic controller, which, in turn, adjusts the current (typically by applying some control voltage at the input of a power amplifier).

An important advantage of the arrangement shown in Figure 1 compared to Figure 2, or any other type of magnetic bearings, is that the current $I$ can be adjusted so that the object deflection from the desired position would remain as zero regardless of the value of the external force acting on the object (the external force has to be static or slowly changing and not to exceed the load limit of the actuator). In effect, this is equivalent to a spring as in Figure 2 with an infinite positive stiffness. 


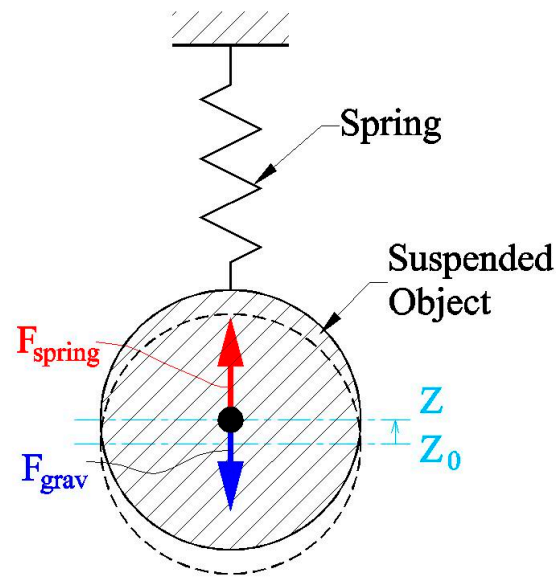

Figure 2. Classical "Mass-Spring" system exhibits positive stiffness, resulting in a stable contact suspension (whereas the interaction between an electromagnet with a constant current and a ferrous object is characterized by a negative stiffness, which makes it unstable without additional controls). $F_{\text {spring: }}$ force produced by the spring.

Since the interaction force between an electromagnet and a ferrous object is always an attractive one, the AMB shown in Figure 1 can operate only if the external forces acting on the object (such as the gravity force $F_{\text {grav }}$ in Figure 1) are directed away from the electromagnet. If the external force can change sign, two electromagnets located on the opposite sides of the object along the force line can be used as shown in Figure 3. For example, if the external force is applied in the positive $Y$ direction, the bottom electromagnet in Figure 3 can be energized to counteract that force and keep the object in place. Alternatively, if the external force is applied in the negative $Y$ direction, the top electromagnet can be energized to counteract it.

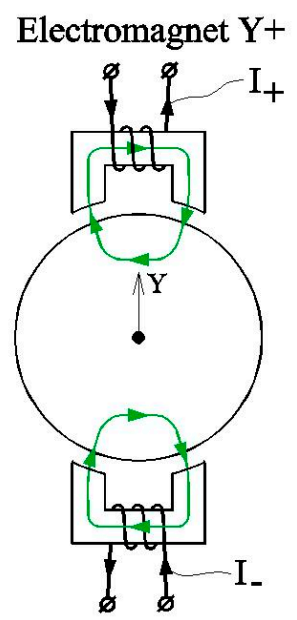

Electromagnet $\mathrm{Y}$ -

Figure 3. An example of a bi-directional electromagnetic actuator.

Situations when the external force acting on an object does not have a dominant constant component, and even may reverse sign, make the current control in an AMB more difficult than when the external force (such as the gravity force in Figure 1) maintains the same direction and never becomes zero. To understand these complications, we need to look at how the force exerted by the electromagnet on the object depends on the current in the electromagnet. This dependence is quadratic in nature: $F_{e m} \sim I^{2}$. Figure 4 shows an example of $F_{e m}$ vs. $I$ curve. Note that the slope of this curve is not constant and changes with the current. Especially important is that when we try to generate 
a small force close to zero, the force is not responding much to changes in the current. This is what makes choosing the right current to offset an external force and stably levitate an object at the desired position difficult.

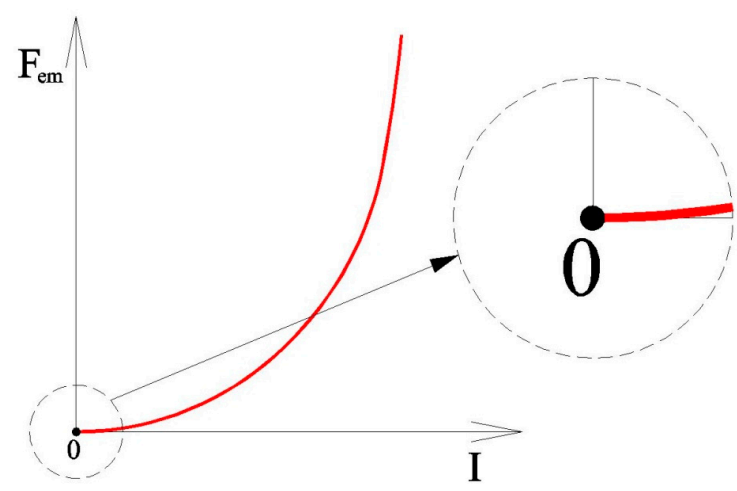

Figure 4. Electromagnetic interaction force $F_{e m}$ between an electromagnet and a ferrous object as in Figure 1 is a non-linear quadratic function of the current in the electromagnet $I$.

A common method used to avoid complications with non-linearity of the $F_{e m}$ vs. I curve, and especially with its low slope (low gain) at small currents, is to pre-energize both electromagnets $Y_{+}$ and $Y$ - in Figure 3 with the same constant current $I_{0}$, called bias current. If the object in Figure 3 is centered between two identical electromagnets and both are energized with the same current, the net pull force on the object would be zero (the bottom electromagnet would pull the object down with exactly the same force as the top electromagnet would pull it up). Therefore, just having a bias current $I_{0}$ by itself does not result in any net force on the object.

In order to produce a force, an additional current, called control current $I_{\mathcal{C}}$, is injected into both electromagnets in such a way that if it adds to the bias current in one coil, it subtracts from it in the opposite coil. For example, in order to produce a force in the positive $Y$ direction, the control current $I_{c}$ has to be added to the bias current $I_{0}$ in the top electromagnet and subtracted from it in the bottom electromagnet. (In practice, there are often two separate coils wound around ferrous core of each electromagnet-one for the bias current and one for the control current.) The force produced by the top electromagnet would be:

$$
F_{T} \sim\left(I_{0}+I_{C}\right)^{2}
$$

and the force produced by the bottom electromagnet would be

$$
F_{B} \sim\left(I_{0}-I_{C}\right)^{2}
$$

The net force exerted on the object would be

$$
F=F_{T}-F_{B} \sim\left(I_{0}+I_{\mathcal{C}}\right)^{2}-\left(I_{0}-I_{\mathcal{C}}\right)^{2}=4 I_{0} I_{\mathcal{C}}
$$

If the bias current $I_{0}$ is kept constant, the control current magnitude is the same on the top and bottom, and the object is kept at the center between two electromagnets, the electromagnetic force exerted on the object would simply be a linear function of the control current (Figure 5). Reversing the sign of the control current will reverse the sign of the force. 


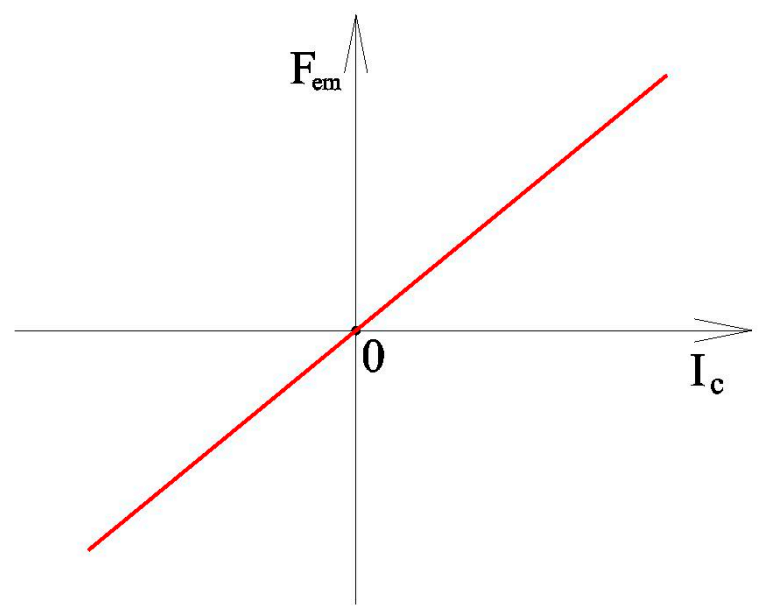

Figure 5. Using bias fields in bi-directional actuators such as in Figure 3 allows linearization of the net electromagnetic force $F_{e m}$ vs. the control current $I_{\mathcal{C}}$.

If two more electromagnets are added as shown in Figure 6, the object position can be controlled along two axes- $X$ and $Y$. The structure shown in Figure 6 represents a typical radial AMB basic design employed in most commercial magnetic bearings. The only difference is that in practical designs, individual electromagnets are often linked together by a common ferrous path as illustrated in Figure 7 with various pole arrangements available.

The structure shown in Figures 6 and 7 would work fine to support a steady ferrous object, but in real-life AMB applications in turbomachinery the object normally spins about its axis $Z$ (directed out of page in Figures 6 and 7) and this brings other complications. To discuss those complications, we will focus only on the bias field for now (for simplicity) and use Figure 8 instead of Figure 6 with the arrows showing the field direction in Figure 6 being replaced with the "North" and "South" designation for the bias electromagnet poles in Figure 8. (The convention is that the magnetic field comes out of the 'North' Pole and returns into the "South" pole).

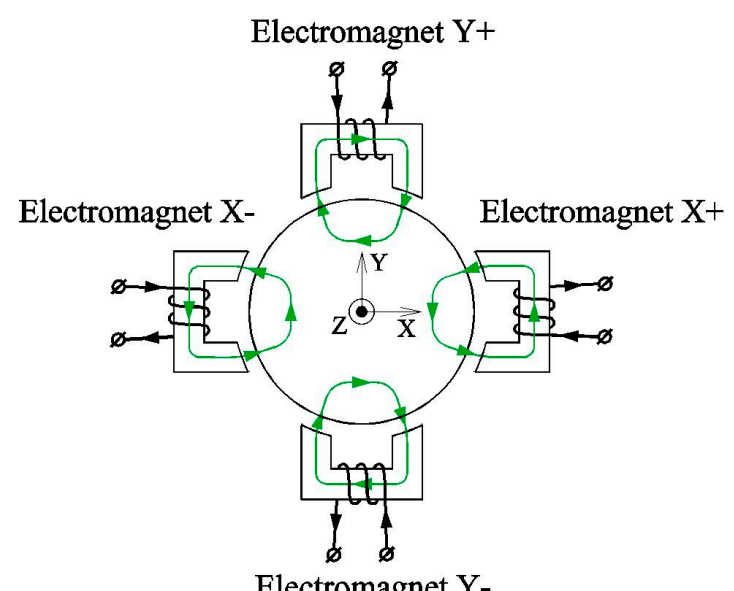

Figure 6. An example of a complete radial actuator capable of producing forces in any radial direction. 


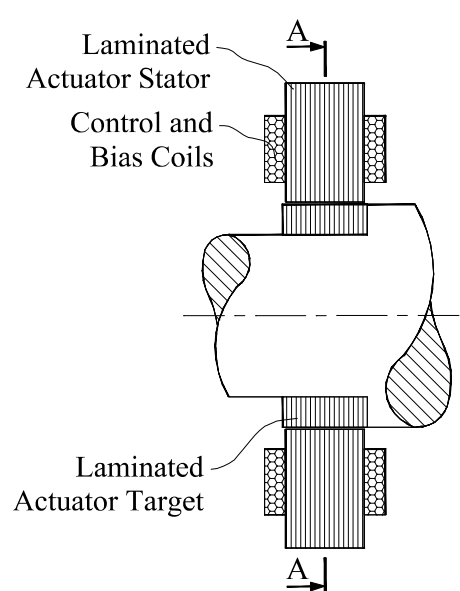

(a)

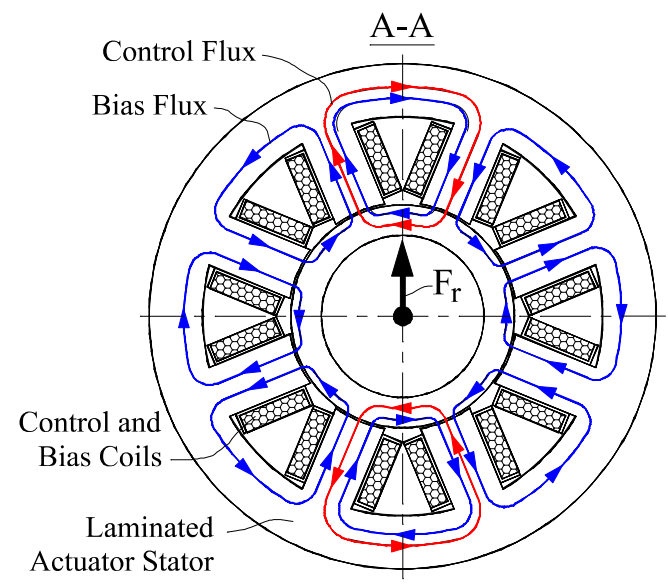

(b)

Figure 7. In commercial radial AMB actuators, continuous laminations with multiple poles are used instead of individual electromagnets as in Figure 6. (a) Axial cross-section of a typical radial AMB actuator; (b) Radial cross-section and magnetic flux paths.

Two symmetric patterns of the bias poles are possible as shown in Figure 8a,b. In either case, a point on the object (rotor) will see changes of the bias magnetic field polarity when the rotor spins: NN-SS-NN-SS in case of Figure 8a and N-S-N-S-N-S-N-S in case of Figure 8b. A complication arises when the rotor material is not only magnetically permeable but also electrically conductive. This is normally the case in practical rotating machines because rotors spinning at high speeds experience high mechanical stresses. While some materials are magnetically permeable but not electrically conductive, such as ferrites, they are not sufficiently strong mechanically. These considerations make magnetically-permeable ferrous steels a material of choice for the rotors, and they are electrically conductive.

When a rotor spins in a non-uniform magnetic field, such as shown in Figure 8a,b, each point of the rotor sees time-periodic magnetic field variations. In Figure 8a, the frequency of the main field harmonic will be $2 \mathrm{f}$ and in Figure $8 \mathrm{~b}$ it will be $4 \mathrm{f}$, where $\mathrm{f}$ is the frequency of rotor rotation in revolutions per second. According to Faraday's law, these periodic changes of the magnetic field cause periodic voltages, which, in case of conductive rotors, cause eddy currents in the rotors.

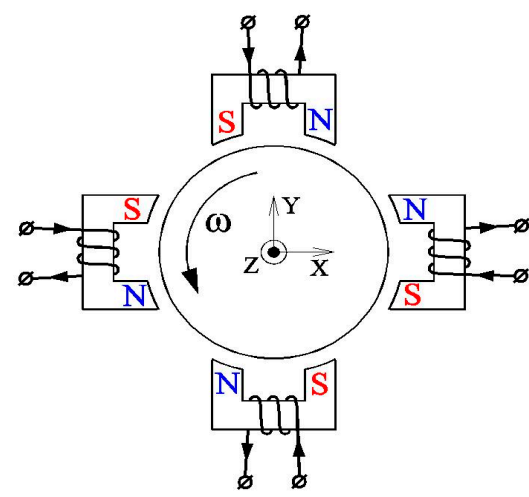

(a)

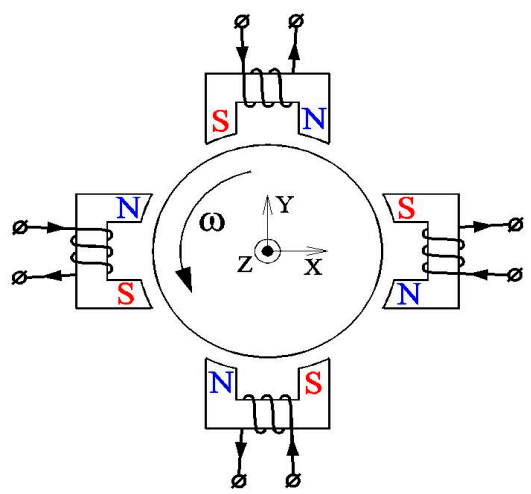

(b)

Figure 8. Two sequences of the bias pole polarities used in heteropolar radial AMB actuators: (a) NN-SS-NN-SS; (b) N-S-N-S-N-S-N-S.

One of the unpleasant outcomes is that eddy currents generate heat in the rotor and cause mechanical drag. 
The other outcome is even more unpleasant. As with any other electrical current, eddy currents induced in a spinning rotor produce their own magnetic field, which gets superimposed on the external magnetic field. The end result is known as a skin effect-a net magnetic field gets expelled from most of the rotor into a very thin (skin-deep) layer on the surface of the rotor [2]. The thickness of this layer can be estimated using the Equation (4) below:

$$
\delta=\sqrt{\frac{1}{\pi \mu_{0} \mu_{r} \sigma} \frac{1}{f_{e}}}
$$

where $\mu_{r}$ and $\sigma$ are relative permeability and conductivity of the rotor material, respectively, and $f_{e}$ is an equivalent electric frequency, which is equal twice the rotor spin frequency for the arrangement shown in Figure 8a and four times for the arrangement shown in Figure $8 \mathrm{~b}$.

For example, electrical silicon steel has $\mu_{r} \approx 4500$ and $\sigma \approx 2.5 \times 10^{6} \mathrm{~S} / \mathrm{m}$. If the rotor has a bias pole arrangement shown in Figure 8a and spins at 30,000 RPM $\left(f=500 \mathrm{~Hz}, f_{e}=1000 \mathrm{~Hz}\right)$, the skin depth would only be $0.15 \mathrm{~mm}$. Since the magnetic flux density in ferrous materials is limited by material saturation to approximately $1.5 \mathrm{~T}$ for regular steels and about $2.1 \mathrm{~T}$ for cobalt alloys, very limited magnetic flux will be able to flow in this thin surface layer. In other words, the bias flux, and similarly, the control flux, will be expelled from the rotor and the actuator will cease to function at speed.

In order to avoid expelling the magnetic flux from a spinning rotor, rotor portions interacting with stationary parts of AMB actuators are normally made out of electrically insulated steel laminations stacked in the axial direction. The electrical insulations between the laminations break the eddy current paths. Note that there will still be eddy currents within each lamination resulting in some decrease of the load capacity of the radial actuator (and, therefore, the bearing) once the skin depth calculated using Equation (4) becomes smaller than the lamination thickness.

The radial actuators illustrated in Figures 6-8 are called heteropolar because the polarity of the bias poles changes around the rotor circumference. This is a consequence of the magnetic fluxes being constrained to the planes normal to the rotation axis (magnetic flux lines always form closed loops-if they exit at one pole they always will have to come back in the other, and in the case of heteropolar actuators both poles lie in the same plane).

There exists another type of actuators for AMBs called homopolar, in which, as the name suggests, the polarity of the bias poles remains the same around the rotor circumference. This is possible because the bias magnetic flux lines lay in the planes aligned with the rotational axis. An example of such an actuator most commonly cited in the literature is shown in Figure 9 [1].

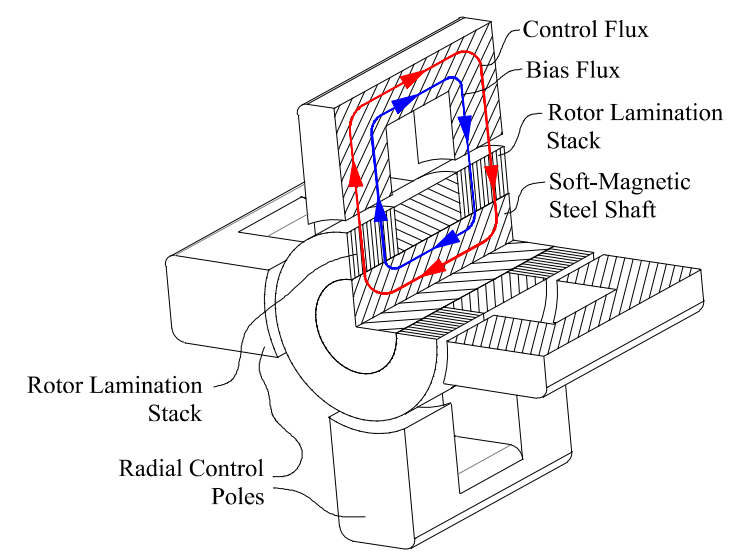

Figure 9. An example of a homopolar radial AMB actuator most commonly cited in the literature.

Figure 9 shows that the magnetic fluxes in the rotor move radially in the rotor lamination stacks before travelling axially in the non-laminated soft-magnetic steel shaft. This illustration will indeed be 
close to reality in this particular construction because the magnetic fluxes will be reluctant to travel axially within the lamination stacks, where they will have to cross multiple non-magnetic layers of insulation between laminations. For example, if the rotor lamination stack is $5 \mathrm{~cm}$ long and the stacking factor (the percentage of the iron in the stack) is $95 \%$, the net thickness of the lamination insulation layers will be $(1-0.95) \times 5 \mathrm{~cm}=0.25 \mathrm{~cm}$, which produces a magnetic reluctance often bigger than the reluctances of the air gaps separating the actuator poles from the rotor.

If the shaft in Figure 9 were non-magnetic, the actuator still would be possible to use by making the spacer separating the two rotor lamination stacks magnetic. The flux in this case, however, would have to travel axially inside the rotor lamination stacks and would have to cross the lamination insulation layers, which would require a larger number of Ampere-turns in the actuator coils.

Because the bias pole polarities in this actuator do not change around the actuator axis in any given axial plane, the rotor will see significantly smaller variations of the magnetic field when spinning compared to the heteropolar actuator design per Figures 6-8 (there still will be some field variations because of the gaps between poles where magnetic fields are much smaller than under the poles). Consequently, in the absence of an external radial load, there will be less eddy currents induced in the rotor at speed. Even when the radial load is present and the magnetic bearing reacts by inducing some control currents and associated control fluxes in the actuator to counteract that load, the effective electrical frequency of the magnetic field changes seen by the rotor will be less than in the heteropolar design.

On the other hand, the heteropolar designs shown in Figures 6-8 will perform better than the design of Figure 9 when reacting to time-varying radial loads. Because the control flux in the heteropolar design is flowing in the lamination plane, the eddy currents it would induce when changing in time would have to flow perpendicular to the lamination plane, where the layers of insulation between the laminations would impede them. In general, for the electrical laminations to be effective, the time-varying magnetic flux lines have to lie within the lamination plane. In this case, the insulation layers between laminations would be in the way of the eddy-currents that otherwise would be induced in the planes perpendicular to the magnetic flux lines in accordance with Faraday's law.

On the contrary, since the portion of the control flux path in the homopolar design shown in Figure 9 goes perpendicular to the lamination planes, the eddy current it would induce when changing in time would flow unobstructed either in solid steel or within the lamination planes. These currents would produce their own magnetic fields countering the original control magnetic field and expelling it from the bulk of the magnetic material due to the skin effect, similar to how it was happening in heteropolar actuator targets at speed. As a result, the dynamic load capacity of the homopolar actuator shown in Figure 9 would be worse than that of the heteropolar actuators. In addition, eddy current would introduce a phase lag between the control current and the control magnetic flux, and, consequently, between the control current and the force produced by the actuator.

An additional drawback of the design shown in Figure 9 is that it is much more complex to manufacture than the heteropolar design shown in Figures 6-8. The heteropolar designs effectively consist of stationary and rotating lamination stacks with electrical coils wound around the stationary poles. The individual laminations are stacked along the actuator axis. This structure has a lot in common with more established electric motors. On the contrary, the laminated poles in Figure 9 are stacked in circumferential directions and practical issues such as forming pole curvatures on their inner diameters, aligning pole and mounting them to the stationary support structure all complicate the actuator design and assembly.

In addition to the radial support, a rotor in a typical rotating machine also needs to be supported axially. If a fully non-contact support is desired, this is typically achieved by using an axial active magnetic bearing, which operational principle is identical to that of the radial active magnetic bearings described above: an axial position sensor detects the axial position of a rotor and provides an input for a magnetic bearing controller, which applies an electrical current to an axial electromagnetic actuator with a magnitude and direction needed to keep the rotor in the desired axial position. 
The most common design of an axial actuator is shown in Figure 10 [1]. It consists of two annular electromagnets placed on both sides of an actuator target disk. Each electromagnet has a bias winding and a control winding, or both functions can be combined in one winding. For practical reasons, both the actuator target disks and soft-magnetic cores of the stationary electromagnets are normally made non-laminated, and the axial actuators such as shown in Figure 10 are prone to the same problems caused by eddy currents that were discussed with application to the homopolar radial actuator per Figure 9: loss of load capacity with frequency and a time lag between the control current and the force the actuator produces.

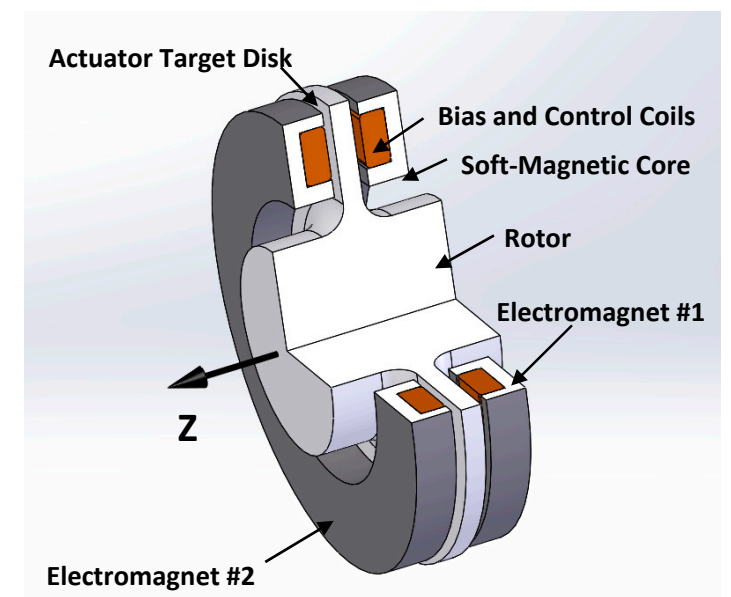

Figure 10. Classic electrically-biased axial actuator for AMBs.

Figure 11 illustrates probably the most common structure of a rotating machine on active magnetic bearings. A rotor with a high length/diameter ratio is radially supported by two radial bearings in $X 1-Y 1$ and $X 2-Y 2$ planes. The bearings are spaced along the rotor axis, which allows controlling two angular degrees of freedom as well. In the axial direction, the rotor is supported by an axial magnetic bearing. When the active magnetic bearings are not energized, the rotor rests on mechanical backup bearings, which also provide support in case of overload or a failure of the magnetic bearings. In the example shown in Figure 11, the rotor is equipped with an impeller on one end as it would be the case with a single-stage air or liquid centrifugal compressor, however, the following discussion will not be limited to the centrifugal compressors only.

Probably in most commercial applications magnetic bearings are used because of their high-speed capability due to the non-contact nature of the rotor support eliminating mechanical wear and tear without the need for lubricants. Therefore, rotors in systems such as shown in Figure 11 are expected to spin fast. It is known that any mechanical structure has some resonance frequencies associated with it, in particular a designer of a rotating machine has to be concerned with bending and torsional resonances of the rotor [3].

Of particular concern are so-called forward-whirl bending modes, because controlling them requires a large amount of damping from the bearings, which, in case of magnetic bearings, would require larger load capacities from the bearing actuators when operating close to the resonance speeds or passing thru them on the way to the operating point. Larger actuator load capacities means larger actuator targets on the rotor, which brings the bending frequencies even lower. In general, the entire machine becomes more compact and easier to design if it is possible to operate below the first forward-whirl bending mode with some margin. Since the actuators are the biggest parts of the entire magnetic bearing system inside the machine, their design has a very large impact on the bending mode frequencies and the choice of the actuator often makes a difference between subcritical and supercritical designs (operating below and above the first forward-whirl bending mode, respectively). 


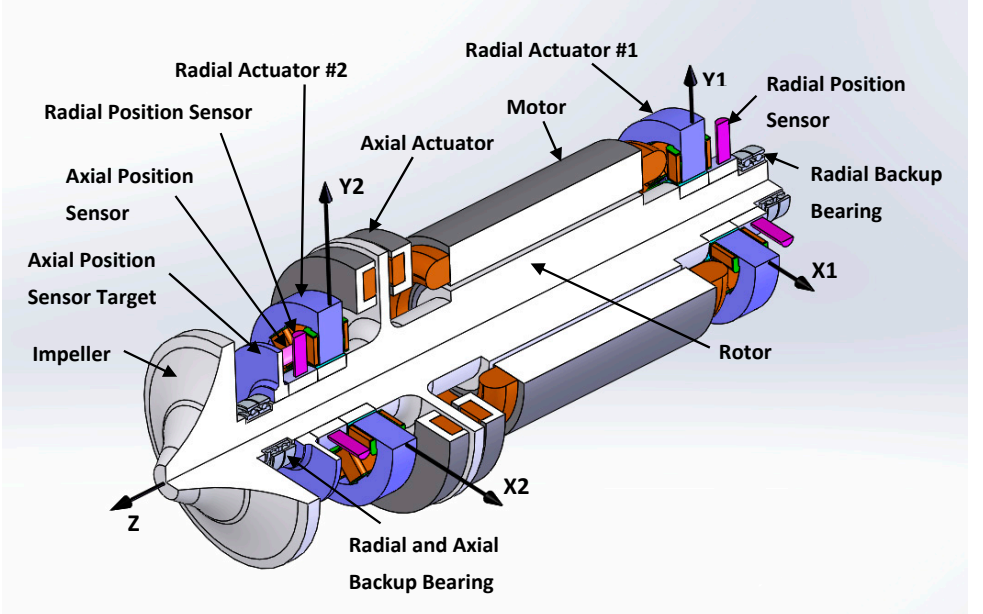

Figure 11. A typical structure of a rotating machine on AMBs.

In summary, the electromagnetic actuators are key components of rotational active magnetic bearing systems and their performance in large degree defines the performance of the entire machine. There is a big variety of the actuator designs out there, and to facilitate the comparison between them, we attempted to list the most important actuator characteristics:

(1) Fitting into a specific rotor design to enable subcritical operation of the machine;

(2) Minimal loss of the actuator load capacity with speed due to the eddy currents;

(3) Minimal loss of the actuator gain and load capacity with frequency due to the eddy currents;

(4) Minimal phase lag between the control current and the force produced by the actuator due to the eddy currents induced when reacting to a dynamic load;

(5) Minimal electrical resistive losses in the actuator target at speed due to the eddy currents;

(6) Minimal actuator negative stiffness;

(7) Minimal aerodynamic drag (for applications not operating in vacuum);

(8) Compactness;

(9) Low cost;

(10) Ease of manufacturing.

\section{Radial Homopolar Electromagnetic Actuators: Design Choices}

\subsection{Separating Bias and Control Magnetic Circuits}

When comparing the homopolar actuator design shown in Figure 9 to heteropolar actuator designs per Figures 6-8, we noticed that the homopolar design per Figure 9 has the advantage of lower eddy-currents induced in the rotor at speed, but generates more eddy currents in the rotor when compensating for the time-varying loads, which lead to the radial force decrease with frequency and a time lag between the control current and the force. The reason was that a portion of the time-varying control magnetic flux in Figure 9 was flowing along the rotor axis, which meant that the rotor had to be laminated so that the lamination plane would be oriented parallel to the rotor axis, which would clearly be impractical.

In another homopolar actuator design shown in Figures 12-14, typical for Calnetix machines, this drawback is overcome by partially separating control and bias flux paths so that only the bias flux, which does not change in time, has an axial component, whereas the control flux is fully contained in the planes of the axially stacked laminations. Figures 12 and 13 show a 2D layout of the actuator and $3 \mathrm{D}$ rendering of the actuator stator, respectively, whereas Figure 14 shows the bias flux path and the control currents and fluxes when generating a radial force along the $Y$ axis. 
The bias flux in the design option shown in Figures 12-14 is generated by a permanent magnet(s), however, it does not necessarily have to be; for example, the magnet(s) in these drawings can be replaced with a soft-magnetic disk to link magnetically the laminated pole assembly and the dead pole, and an electrical coil wound around the actuator axis can be placed inside the disk and used to induce the bias field. We will discuss advantages and disadvantages of each approach later.

As Figure 14 shows, the bias magnetic flux generated by the permanent magnet flows from the magnet through a dead pole towards a soft-magnetic shaft, crosses a radial air gap separating the dead pole from the shaft, flows axially inside the shaft towards the laminated actuator target firmly mounted on the shaft, exits this target radially thru four radial air gaps separating the target from four radial control poles within the laminated radial pole assembly (labeled " 1 " through " 4 " in Figures 12 and 14), and flows within those poles towards the bias permanent magnets where it completes the loop.

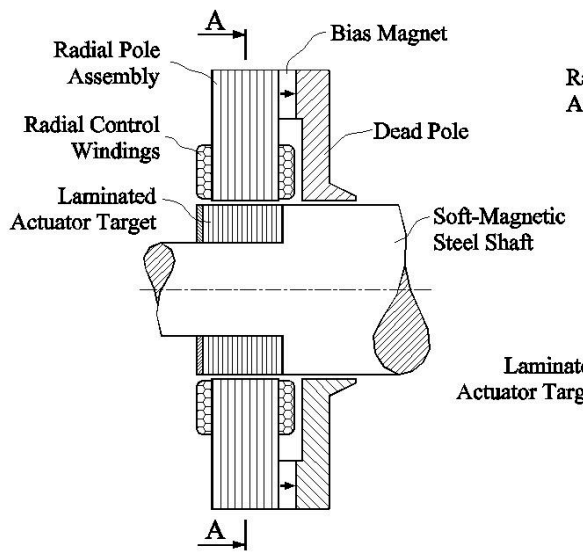

(a)

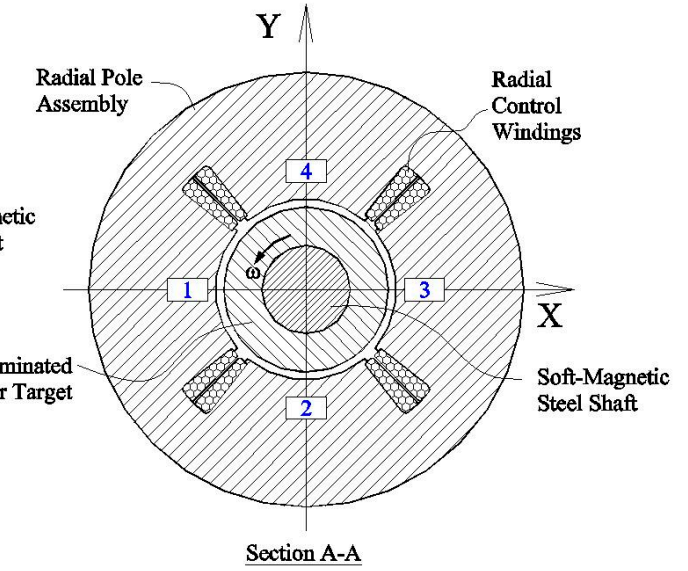

(b)

Figure 12. Construction of the radial homopolar PM (permanent magnet)-biased AMB actuator with partially separated bias and control magnetic circuits. (a) axial cross-section; (b) radial cross-section of the radial pole assembly.

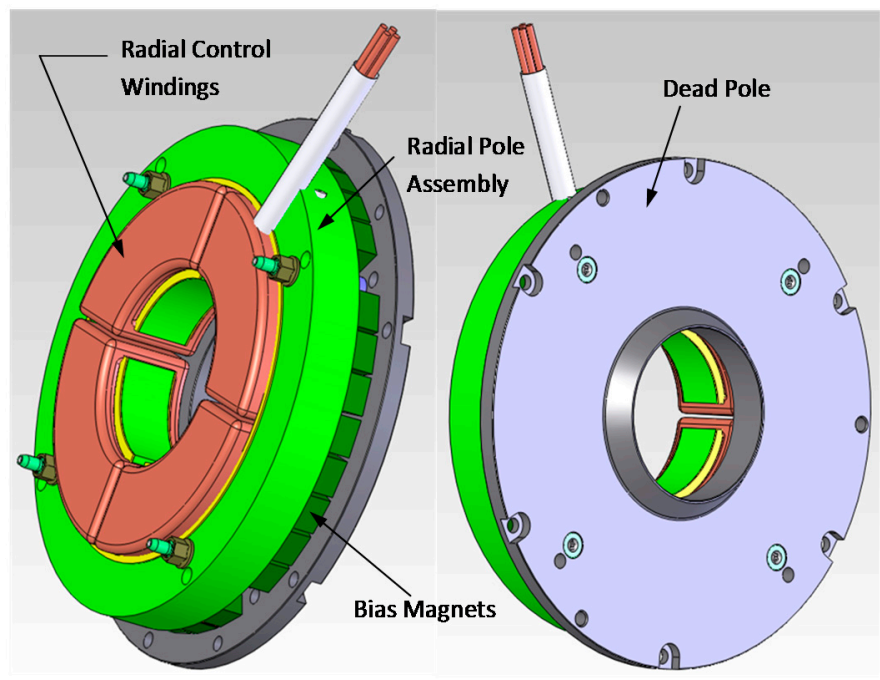

(a)

(b)

Figure 13. 3D rendering of the stator of the radial homopolar PM-biased AMB actuator with partially separated bias and control magnetic circuits. (a) view from the radial control pole side; (b) view from the dead pole side. 


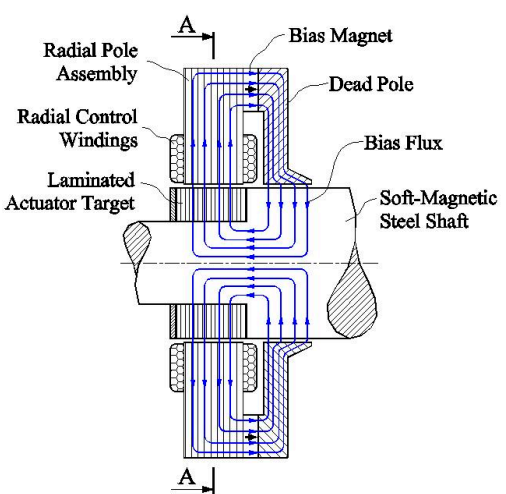

(a)

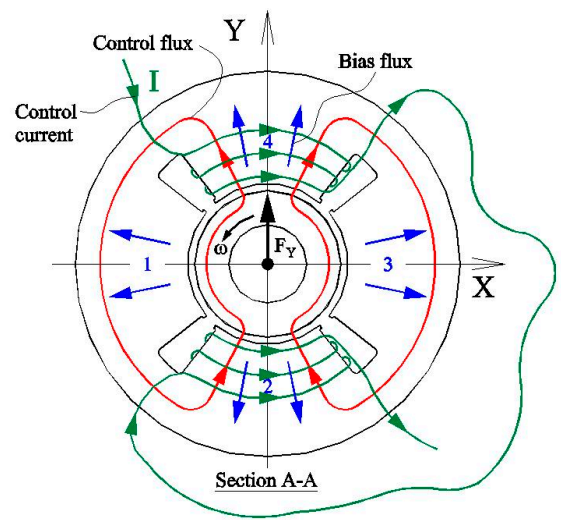

(b)

Figure 14. Operating principle of the radial homopolar PM-biased AMB actuator with partially separated bias and control magnetic circuits. (a) axial cross-section and bias flux path; (b) radial cross-section and illustration of the radial force generation.

In order to generate radial forces, this actuator is equipped with four radial control windings wound around each of the four poles. Each two diametrically-opposite windings are connected in series so that they both produce magnetic fluxes flowing in the same direction.

In the example shown in Figure 14b, two radial control windings wound around poles 2 and 4 are energized with control currents to produce a radial force $F_{y}$ in the positive $Y$ direction. The control flux produced by these two coils (shown in red) adds to the bias flux (shown in blue) in the upper radial air gap under the pole \#4, but subtracts from it in the lower air gap under the pole \#2, resulting in a higher flux density in the upper air gap than in the lower, and, consequently, a radial force $F_{y}$ directed upwards. Applying a current in a similar manner to coils \#1 and \#3 (coils not shown for clarity) will produce a force in the $X$ directions and a combination of two currents allows generating a force in any direction in the radial plane.

Note that this design offers the main benefit of the homopolar design - the polarity of the bias field in the poles does not alternate around the rotor, but it is also free of the main drawback of the design shown in Figure 9-time-varying magnetic fields do not flow axially. It can also be noticed that this design is easier to put together than the design in Figure 9, even though it is still more complicated than the heteropolar design in Figures 6-8.

In some cases, a version of the design shown in Figure 15 can be used in which the bias magnetic flux returns not through a "dead" pole but through a second radial pole assembly. This design version may better utilize the surface area of the rotor because the rotor surface under the dead pole in Figures 12-14 does not contribute to the "useful" controllable force, only to the negative stiffness. However, this advantage, even if it exists, may not be a big one because the dead pole thickness at some distance from the rotor may be small enough to saturate the dead pole material, which also brings two additional advantages:

(1) The bias magnetic flux becomes less dependent on the temperature because it is controlled (to some extent) by the saturation flux density of the dead pole material, which does not change with temperature nearly as much as the magnetic flux emanated from the magnet.

(2) The contribution to the negative stiffness from the dead pole decreases because the total flux under the dead pole does not increase as much when the rotor deviates from the centered position as it would if the dead pole was not saturated. The negative stiffness can be reduced even further if the dead pole is made axially longer in close proximity of the rotor as shown in Figures 12-14 because this lowers the flux density in the air gap between the dead pole and the rotor [4]. The axial extension to the dead pole in most machines fits conveniently under the endturn of a nearby motor, thus not adding any extra length to the rotor. 
Similar to Figures 9, 14 and 15 illustrate the magnetic flux path in the rotor assuming the shaft under the rotor laminations is magnetically permeable, in which case the flux tends not to travel axially within the lamination stacks to avoid crossing high magnetic reluctances associated with the non-magnetic insulation layers between the laminations. If the shaft were non-magnetic, the actuators still could be used, but the bias magnets would have to be larger to account for the higher magnetic reluctances associated with moving axially across the lamination stacks.

An additional complication in designs where the magnetic flux travels across the lamination stacks is that the stacking factors are typically not very consistent, resulting in variations of the reluctances associated with axial paths across lamination stacks, and, subsequently, variations of the bias levels in the actuators. Once again, in the design shown in Figure 14, this can be mitigated to some degree by saturating the dead pole. In general, however, using a soft-magnetic shaft under the rotor lamination with a sufficient cross-section to carry the entire bias flux axially is a preferred approach, whenever feasible.

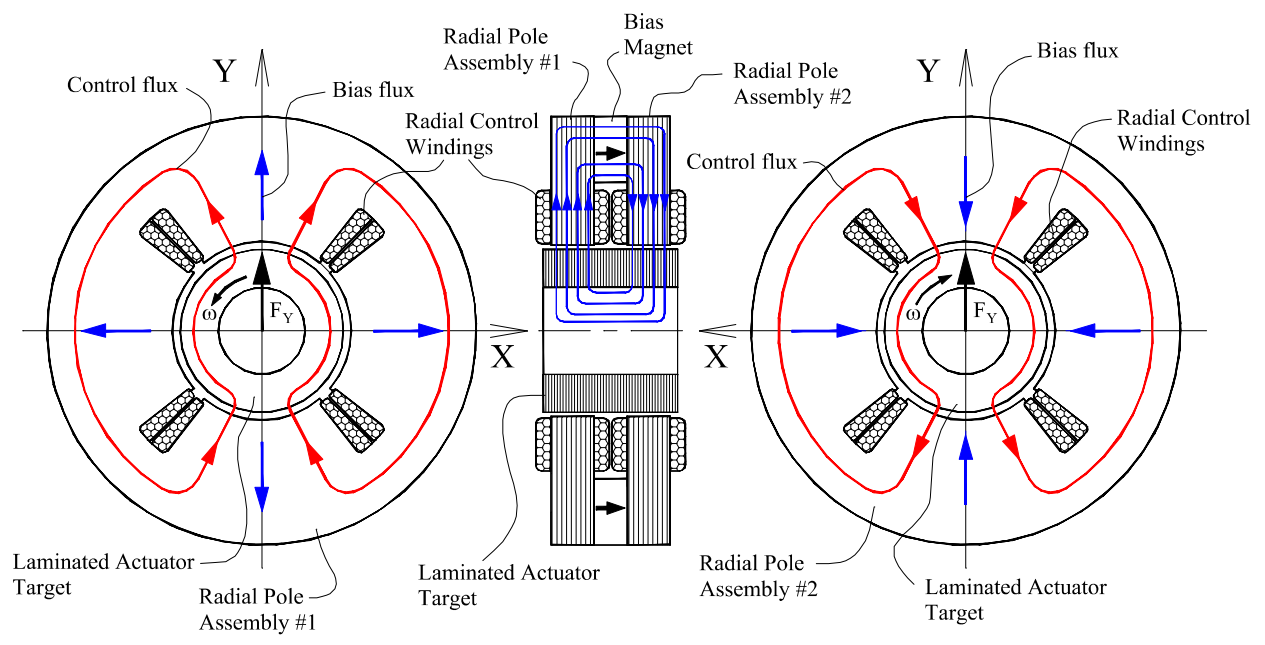

Figure 15. A version of the radial homopolar PM-biased AMB actuator with partially separated bias and control magnetic circuits and two radial control pole assemblies.

\subsection{Using Permanent Magnets to Generate Bias Flux}

Earlier we have mentioned that this type of actuators does not necessarily require permanent magnets to generate the bias flux, which can also be generated by a coil with a current. There are both advantages and disadvantages of using permanent magnets, which are listed below.

Advantages:

(1) For the same amount of the bias flux generated in a magnetic circuit with reluctance typical for magnetic bearings, permanent magnets end up being much smaller than coils.

(2) Using permanent magnets in the bias circuit results in significantly smaller negative stiffness than when using coils due to the large internal reluctance of the magnets (see [5] for details).

(3) Fewer wires between the machine and the magnetic bearing controller and fewer feedthroughs on the machine side (especially important when the machine has to be hermetically sealed).

(4) No amplifier (current source) is needed for the bias circuit.

(5) No power dissipation in the bias circuit.

Disadvantages:

(1) Bias flux may change with temperature unless preventive measures such as saturating the dead pole were taken. In practice, however, the change of the bias flux with temperature in this type of bearings does not greatly affect the bearing controllability, but does reduce gain and load capacity, which needs to be taken into account at the design stage. 
(2) Magnets cannot be "turned-off", which complicates the assembly.

(3) Magnets are more expensive than coils.

While many electromagnetic actuator designs exist that utilize permanent magnets for bias, the important advantage of the designs shown in Figures 12-15 is that the control magnetic flux generated by the control coils does not go through the magnets. The advantages resulting from this design feature include:

(1) Much smaller control coils-should the control flux go through a magnet, which has relative permeability very close to one, the additional reluctance of the magnet would require a very high number of additional Ampere-turns in a coil to maintain the same flux level.

(2) No risk of demagnetizing the magnet with the control magnetic field. This risk becomes especially high in designs where the control field does go through the magnet and, in the attempt to minimize the increase of the control coil size due to the additional magnet reluctance (see the first item above), the magnets are made thin with a large surface area (to maintain the needed bias flux level).

\subsection{Combination Radial/Axial Homopolar PM-Biased Actuators}

Contrary to the radial actuators used in active magnetic bearing systems, axial actuators are pretty much always homopolar (see Figure 10 for example). This is primarily because it would be more difficult to make them heteropolar, while there would be no obvious benefits. We found, however, that most often an alternative solution-a combination radial and axial homopolar PM-biased electromagnetic actuator-is a better fit for a practical industrial magnetic bearing application. The references [5,6], for example, provide a comparison of the overall performance of compressor systems designed with such combination radial/axial actuators and a more traditional arrangement of a separate electrically-biased axial actuator as in Figure 10 and a heteropolar radial actuator as in Figure 7.

The two main combination actuator designs manufactured by Calnetix are schematically shown in Figures 16 and 17. We will refer to the earlier design shown in Figure 16 as a "symmetric" combination actuator because of its symmetry about the middle plane [7]. We normally refer to the other design, shown in Figure 17, as the "side-by-side" combination actuator because its radial and axial control magnetic circuits are arranged side by side along the actuator axis [8,9].

Figure 16 shows the design and illustrates the operational principle of the "symmetric" combination actuator. The bias flux in this design is generated by two sets of permanent magnets located on both sides of the laminated radial pole assembly with identical magnet poles oriented towards that assembly (In principle, only magnets on one side can be used, but normally there is no practical advantage in doing so). The other permanent magnet poles are coupled to the axial poles. Both radial and axial channels of the actuator share the same actuator target mounted on the rotor. The actuator target is laminated along the rotor axis. The bias fluxes (shown in blue lines) generated by the magnets enter the axial poles, travel towards the rotor, cross the axial air gaps and enter the actuator target axially. Within the actuator target, two bias fluxes change their directions from axial to radial and join together. They exit the actuator target radially across the radial air gaps separating the radial poles from the target, enter the radial poles and travel towards the permanent magnets where they close the loops.

If the axial control coil is energized with a current as shown in Figure 16a, an axial control magnetic flux (shown in red) will be induced flowing in a loop consisting of two axial poles, the axial backiron, the actuator target and two axial air gaps separating it from the axial poles. In the example shown in Figure 16a, the control flux adds to the bias flux in the left axial air gap, but subtracts from it in the right axial air gap, resulting in the higher flux density on the left, and, consequently, a net electromagnetic force pulling the actuator target to the left. Reversing the control current direction would reverse the direction of the force. Note that the axial control magnetic flux will not enter the 
radial pole assembly due to the system symmetry, and, therefore, no significant axial-to-radial cross coupling should occur in this design.

The operation of the radial channel, illustrated in Figure 16b, is fully identical to the operation of the pure radial actuator illustrated earlier in Figure 14.

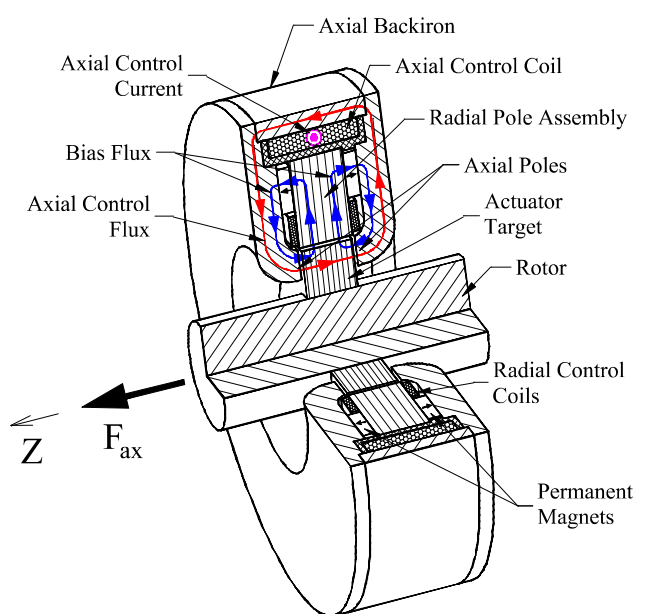

(a)

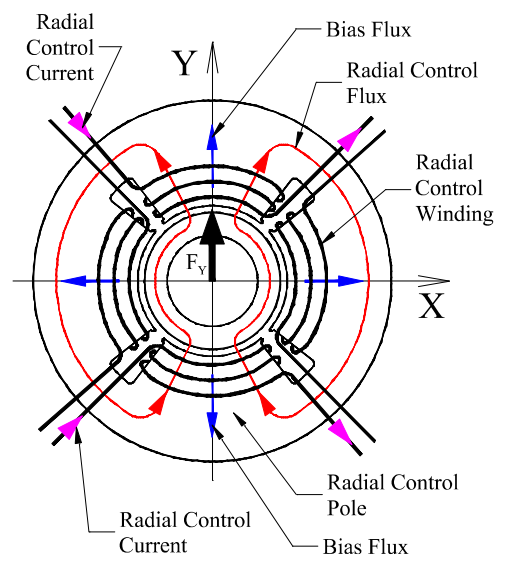

(b)

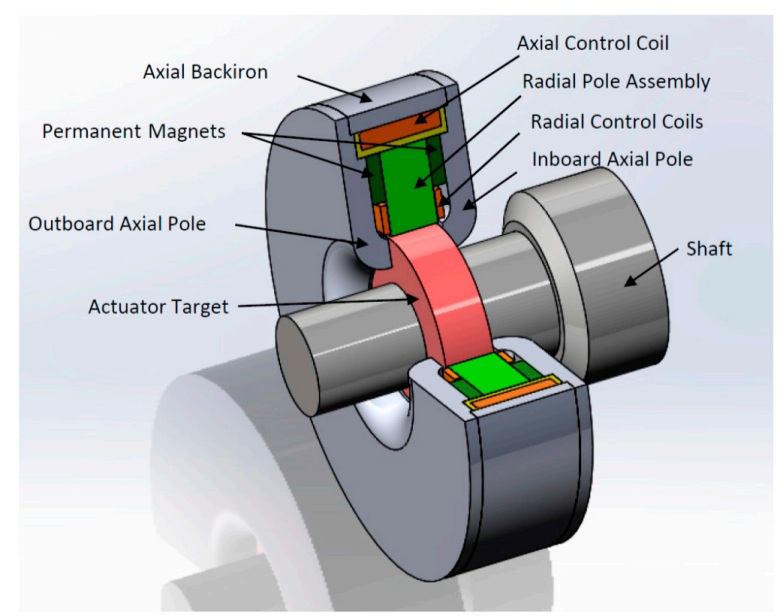

(c)

Figure 16. "Symmetric" homopolar PM-biased combination radial/axial AMB actuator. (a) Axial cut-out section and axial channel operating principle; (b) Radial lamination cross-section and radial channel operating principle; (c) 3D rendering.

In the other version of the combination actuator, shown in Figure 17, the permanent bias magnet is located only on one side of the radial pole assembly and coupled to the closest axial pole. In contrast to the "symmetric" design, the other axial pole is located not on the opposite side of the radial pole assembly, but on the same side just further away from it axially. This design features two actuator targets on the rotor: one is a non-laminated axial target and the other is a laminated radial target. The two targets must be coupled magnetically through either a soft-magnetic shaft (in which case the axial target is normally made as a part of the shaft), or through a soft-magnetic coupler added to the shaft. The bias magnetic flux generated by the magnet splits into two portions between two axial poles (see Figure 17a), these two flux portions then travel towards the rotor and cross the axial gaps before joining back together within the axial actuator target. After that the entire bias flux travels towards the radial actuator target, it changes direction to radial, leaves the radial actuator target through the radial air gap, enters the radial control poles and travels back to the magnet to complete the loop. 
Once again, the operation of the radial channel in this actuator, illustrated in Figure 17b, is fully identical to the operation of the pure radial actuator illustrated earlier in Figure 14.

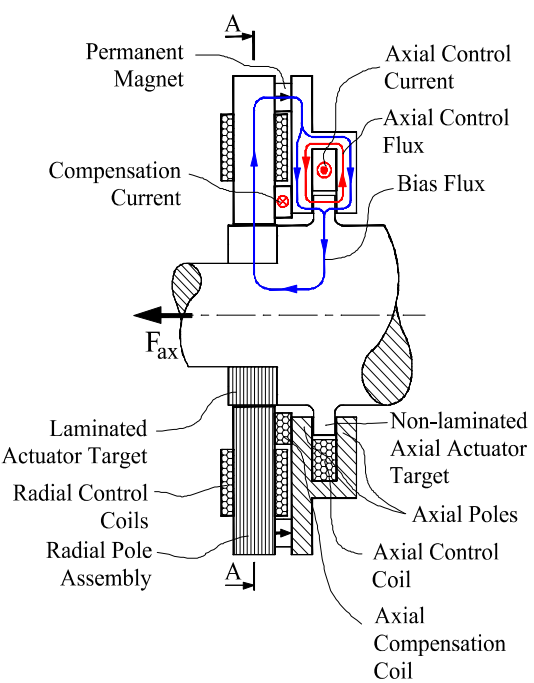

(a)

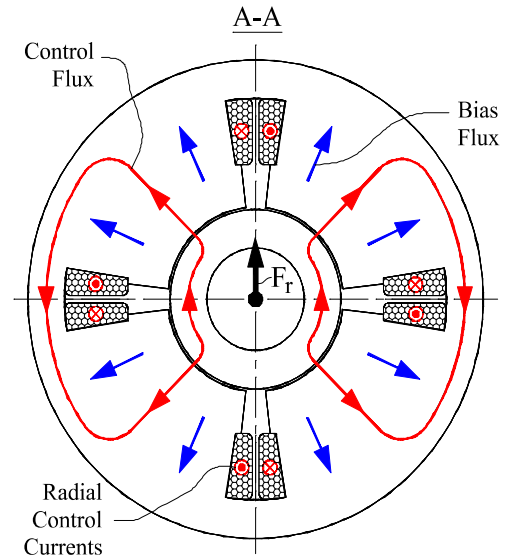

(b)

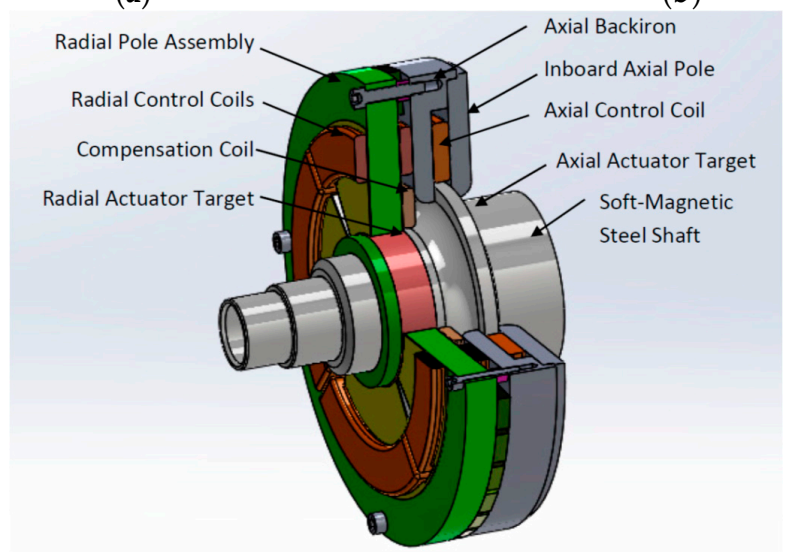

(c)

Figure 17. "Side-By-Side" Homopolar PM-Biased Combination Radial/Axial AMB Actuator. (a) Axial cut-out section and axial channel operating principle; (b) Radial cross-section and radial channel operating principle; (c) 3D rendering.

Operation of the axial channel, however, is a little bit more complicated than in the previous "symmetric" combination actuator design. As before, energizing the axial control coil with a current results in an axial control magnetic flux that flows in the loop consisting of two axial poles, the axial backiron, the actuator axial target and two axial air gaps separating it from the axial poles. Similar to Figure 16, in the example shown in Figure 17, the control flux adds to the bias flux in the left axial air gap, but subtracts from it in the right axial air gap, resulting in higher flux density on the left, and, consequently, a net electromagnetic force pulling the actuator target to the left. Reversing the control current direction would reverse the direction of the force.

However, contrary to the "symmetric" design per Figure 16, this design lacks the symmetry about the axial middle plane and the axial control coil will generate a magnetic flux in the radial pole assembly in addition to the nominal axial control magnetic flux shown in Figure 16, resulting in an axial-to-radial cross coupling. The amount of cross-coupling depends on the clearance between the radial poles and the closest axial pole, and may not be an issue if those poles are spaced far enough [8], but this is not a practical solution for commercial applications requiring the actuator to be as axially 
compact as possible. This complication is overcome by using a "bias flux compensation" coil $[9,10]$ shown in Figure 17.

In more detail, because of the lack of the symmetry in this design discussed earlier, in addition to the axial control flux path shown in Figure 17a, there also exists an alternative path for the magnetic flux induced by the axial control current, which leads to the existence of a leakage magnetic flux shown in Figure 18. (From this point on, we will refer to the control flux that would be produced in the absence of the leakage as the nominal control flux.) Note that the leakage flux in Figure 18 is represented by two flux lines-a reason for this will become clear shortly.

Presence of the leakage magnetic flux has two negative consequences:

(1) The radial bias flux changes with the axial control current and (to a lesser degree) with the axial position of the rotor resulting in the axial-to-radial cross-coupling. This is because the leakage flux in Figure 18 adds to or subtracts from the bias flux shown in Figure 17 in the radial air gaps and control poles.

(2) The axial force-vs.-current curve becomes non-linear because the control flux in the right axial pole is always greater in magnitude than the control flux in the left pole due to the added leakage flux.

The bias flux compensation coil is introduced to overcome the negative effects of the leakage flux by generating a compensation magnetic flux, which is also indicated by two flux lines in Figure 18 . In most parts of the magnetic circuit, the compensation flux flows along the same path as the leakage flux but in the opposite direction. The exception is the axial control circuit where the entire leakage flux flows through the right pole, whereas the compensation flux splits: one line flows through the left pole and one through the right (this is why we used two flux lines to represent the leakage and the compensation fluxes).

One can see that in most of the magnetic circuit, except for the two axial poles, there are two leakage flux lines and two compensation flux lines going in opposite directions, and, therefore, cancelling out each other. In the right axial pole, one compensation flux line directed radially inwards gets subtracted from two leakage flux lines going radially outwards, resulting in a single line of the net flux going outwards. In the left axial pole, where the leakage flux did not exist, there will be one line directed radially inwards introduced by the compensation flux. Thus, the result of the superposition of the leakage and compensation fluxes will look exactly as the axial control flux shown in Figure 17. With the right amount of compensation in place, there will be no changes of the radial bias flux and the net control fluxes in the left and the right poles will be the same (and slightly larger than the nominal control flux), as needed for the axial channel linearity. Reference [10] shows how to size the compensation coil as well as some limitations of this approach.

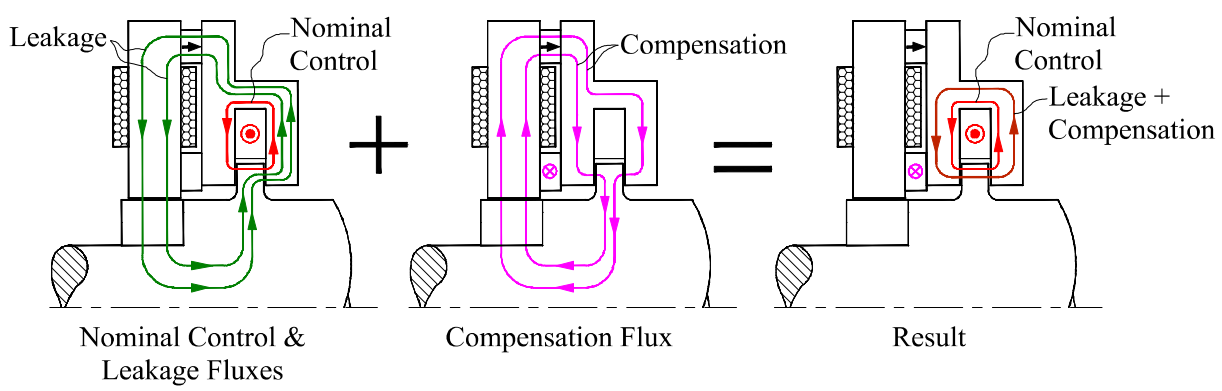

Figure 18. Graphic illustration of the bias compensation principle in the SBS (side-by-side) combination actuator.

Both the "symmetric" and the "side-by-side" designs offer several critical advantages over the conventional arrangement of a dedicated electrically-biased axial actuator per Figure 10 and a radial heteropolar actuator per Figure 7. These include: 
(1) Shorter axial length leading to a more compact design and often to better rotordynamics.

(2) Lower aerodynamic drag due to lower axial actuator target OD (outer diameter), except when the rotor spins in the vacuum.

(3) Lower stresses in the rotor due to lower axial actuator target OD.

(4) Lower part count.

(5) Lower, and nearly constant, negative stiffness.

On top of those common advantages, both designs have their own advantages and disadvantages. Below, we provide a brief summary.

(1) "Symmetric" combination actuator

- Advantages

i. $\quad$ No need for the compensation coil.

ii. Works with non-magnetic shafts (actually it works better with non-magnetic shaft).

iii. The most axially compact design.

iv. Easy integration into a soft-magnetic housing because the entire actuator is already enclosed by soft-magnetic parts (axial poles and axial backiron).

- Disadvantages

i. Limited axial bandwidth due to

a. Long distance the control magnetic flux has to travel thru non-laminated axial poles and axial backiron;

b. Macroscopic currents induced in continuous radial backiron surrounding the axial control magnetic flux flowing in the actuator target.

The issues a. and b. above can be mitigated as described in [11,12], resulting in an improved actuator axial bandwidth , but at the cost of a significant increase of the design complexity.

ii. Limited axial load capacity due to the limited outer diameter of the actuator target (which is limited because the entire actuator target is made laminated and electrical steel laminations have smaller strength than solid steel, especially when they are pre-stressed due to the shrink-fit installation on the shaft).

iii. In some applications with large overhang mass(es) at the rotor end(s) (such as overhang impellers in turbocompressors and turboexpanders), having the reduced shaft diameters on both sides of the actuator target negatively effects (reduces) the first bending mode frequency.

(2) "Side-by-side" combination actuator

- Advantages

i. Good axial bandwidth right from the start without any modifications complicating the design.

ii. Works very well with soft-magnetic shafts.

iii. The gradual decrease of the shaft diameter from the axial actuator portion to the radial actuator portion makes these actuators a perfect fit for turbocompressor/expanders with overhang impellers because this geometry helps increasing the rotor first bending mode frequency. 
- Disadvantages

i. Requires a compensation coil.

ii. If the shaft is non-magnetic, a soft-magnetic coupler has to be added to link magnetically the radial and axial actuator targets.

iii. The actuator OD has to be separated from the housing when the housing is soft-magnetic to avoid magnetic shorting the magnets.

One can note that these two designs are often complementary to each other, for example, the "symmetric" actuator is easier to integrate when the shaft is non-magnetic, whereas the "side-by-side" solution would be a better fit for a soft-magnetic shaft.

\section{Design and Analysis of Homopolar PM-Biased Actuators}

Design and analysis of homopolar PM-biased actuators as in Figures 16 and 17 is significantly more difficult than design of either heteropolar actuators per Figure 7 or the electrically-biased thrust bearing per Figure 10 because of the 3-dimensional magnetic field distributions and numerous leakage paths. Finite Element Analysis (FEA) is pretty much required to achieve a reasonable design accuracy, but FEA by itself cannot be used to design the system, only to analyze the existing designs.

In order to facilitate a rapid design process, we developed an automated approach in which the design process is driven by analytical equations, the parameters of which are constantly adjusted based on FEA results. The analytical portion of the design is based around a magnetic circuit corresponding to a lumped magnetostatic model of the analyzed actuator, which takes into account all the leakage paths that affect the design. For example, Figure 19 shows a lumped magnetostatic model of a "side-by-side" electromagnetic actuator [10], whereas Figure 20 shows a simplified magnetic circuit corresponding to the model in Figure 19 with some parameters in Figure 19 being grouped together as described by the equations below:

$$
\begin{aligned}
& R_{\text {leak_top }}=R_{\text {leak_top1 }}|| R_{\text {leak_top2 }}|| R_{\text {leak_top3 }} ; \\
& R_{\text {leak_ax-rad }}=R_{\text {leak_ax-rad1 } 1}|| R_{\text {leak_ax-rad2 } 2} / 4 \text { (divided by four because there are four poles with } \\
& \text { associated reluctances connected in parallel); } \\
& R_{\text {rad_tot }}=\left(R_{\text {rad }}|| R_{\text {fr_rad }}|| R_{\text {leak_rad1 } 1}\right) / 4 \text {; } \\
& R_{a x 1 \_t o t}=R_{a x 1}|| R_{f r \_a x 1}|| R_{\text {leak_ax1-shaft }}|| R_{\text {leak_ax1-trg_top }}|| R_{\text {leak_ax1-trg_btm }} ; \\
& R_{a x 2 \_t o t}=R_{a x 2}|| R_{f r \_a x 2}|| R_{\text {leak_ax2-shaft }}|| R_{\text {leak_ax2-trg_top }}|| R_{\text {leak_ax2-trg_btm }}|| R_{f r \_a x 2 \text {-shaft }}|| \\
& R_{\text {leak_ax2-shaft_side; }}
\end{aligned}
$$

where symbol I I means parallel connection, for example,

$$
a\|b\| c=\left\{\frac{1}{a}+\frac{1}{b}+\frac{1}{c}\right\}^{-1}
$$

Figure 21 shows a block diagram of the automated actuator design process. The design starts with assuming some initial values for the lumped model parameters using analytical approximations for the leakage reluctances given for example in [13]. Then, the program sizes an actuator, generates an input geometry (along with material properties) for FEA, runs FEA, evaluates if the FEA results meet the design criteria, and, if not, adjusts the analytical parameters based on the FEA outputs, resizes the actuator and repeats the process. Note that the design criteria include not only the actuator performance parameters such as load capacity and negative stiffness, but also levels of saturation for different magnetic material to make sure that they are used to their full capacity.

Even though analyzing the Homopolar PM-Biased Actuators described here is, strictly speaking, a three-dimensional problem, in order to speed up the process it can be reduced to two dimensions using, for example, techniques described in [4]. As a tool for two-dimensional FEA, we found a freeware FEMM [14] particularly easy to use and interface with our software while producing accurate results. 


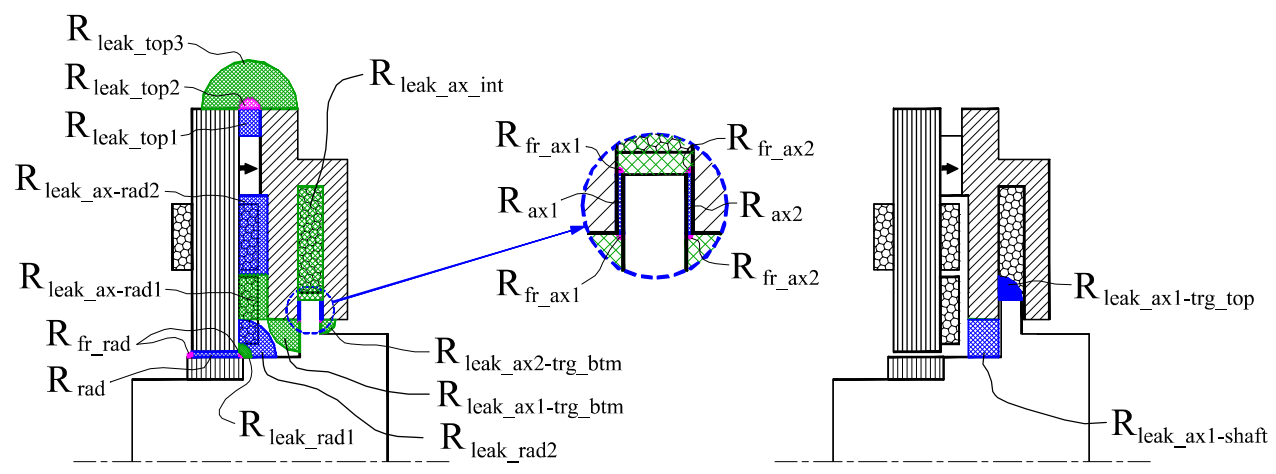

(a)

(b)

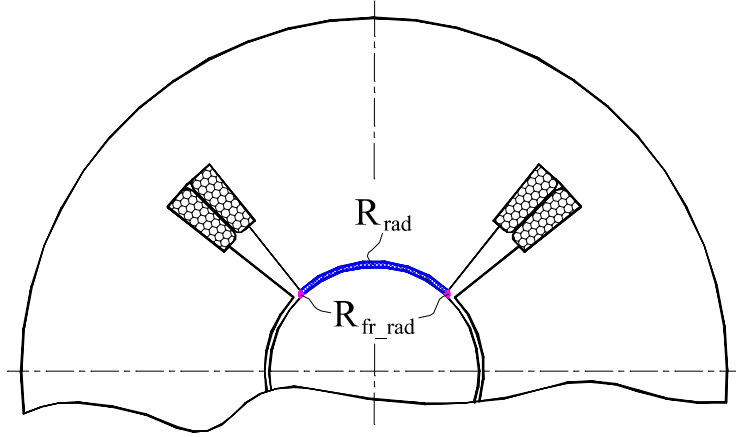

(c)

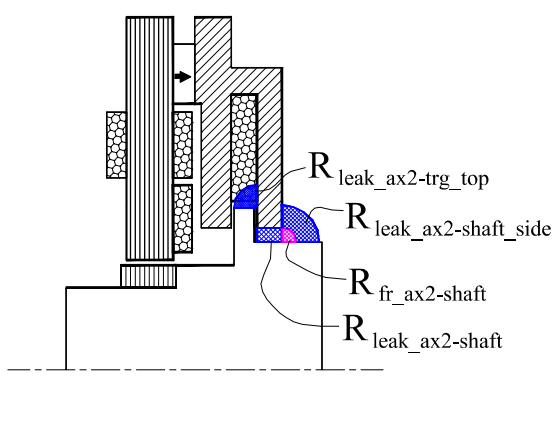

(d)

Figure 19. Lumped magnetostatic model of the "side-by-side" homopolar PM-biased combination radial/axial AMB actuator. Multiple views (a-d) are used to show elementary reluctances to avoid overcrowding.

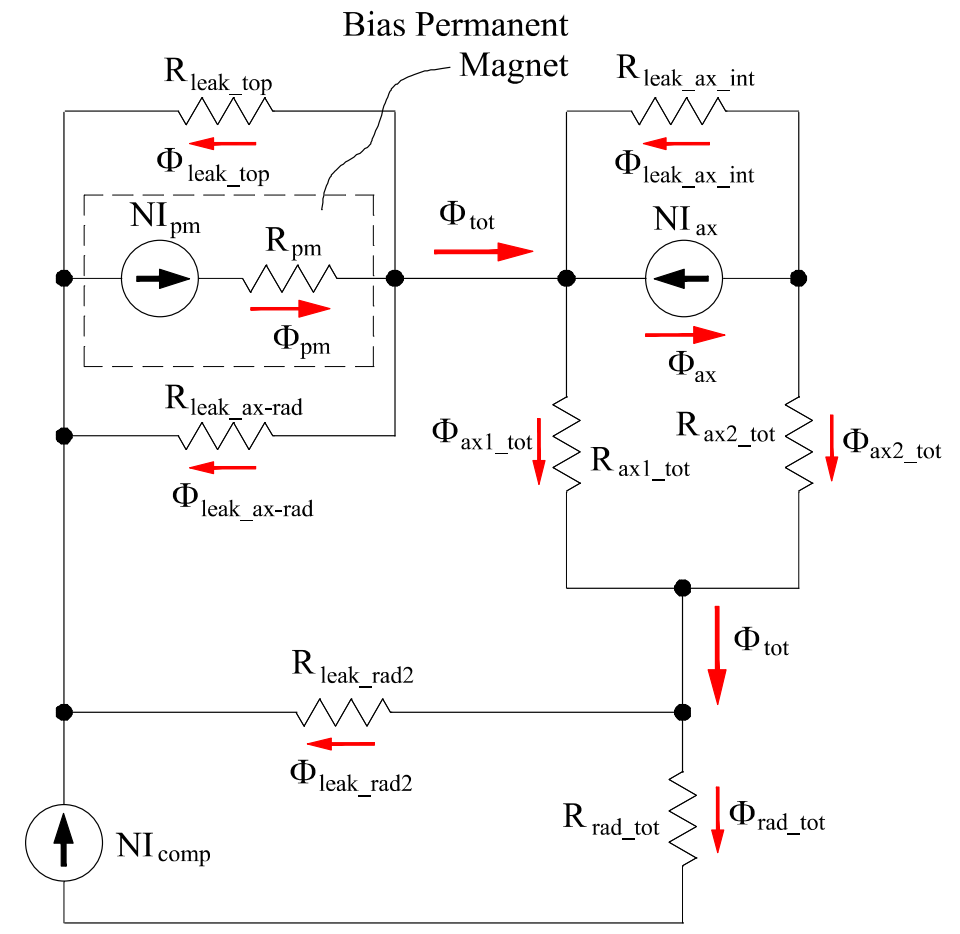

Figure 20. Magnetic circuit for the analysis of the magnetostatic actuator model per Figure 19. 


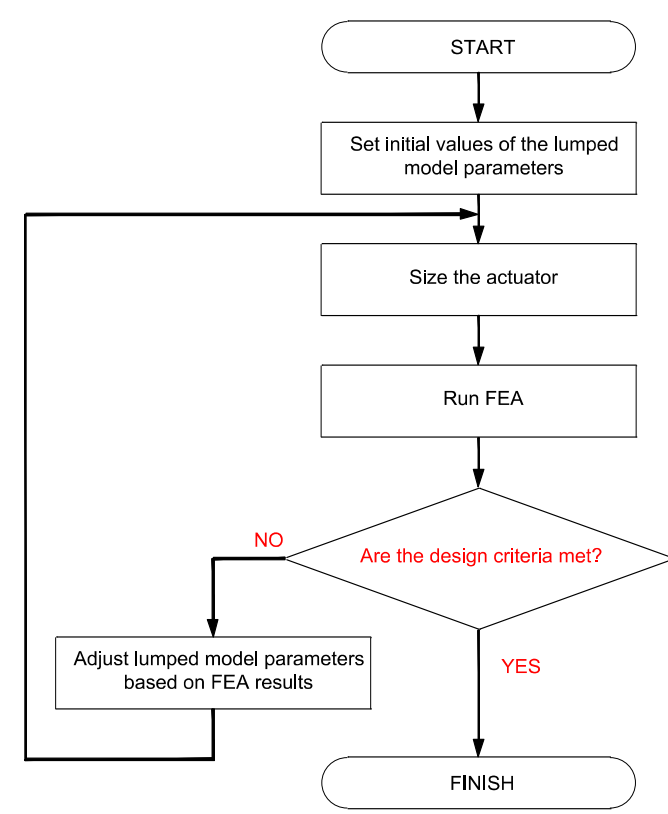

Figure 21. Block diagram of the algorithm for sizing homopolar PM-biased AMB actuators.

\section{Application Examples}

\subsection{Flywheel Energy Storage}

Calnetix's affiliate Vycon has developed a number of flywheel energy storage systems for a variety of applications ranging from $300 \mathrm{~kW}$ uninterruptable power supplies to $500 \mathrm{~kW}$ metro rail energy recovery systems. The basic structure of the flywheel is shown in Figure 22 [15]. It consists of a solid steel flywheel hub firmly connected to a permanent magnet motor/generator. The entire rotor is located vertically and supported by a radial homopolar PM-biased AMB on top and a symmetric combination radial/axial AMB at the bottom. This application is rather easy for magnetic bearings because the rotor does not experience significant dynamic loads but using homopolar PM-biased actuators is important because the rotor almost continuously spins at very high speeds (37,000 RPM) in a vacuum, where the heat extraction is very limited. Therefore, low eddy-current losses in the rotor due to the homopolar design and generating the bias field by permanent magnets without resistive losses become very important advantages. The other important advantage is minimizing the number of wires crossing the vacuum boundary.

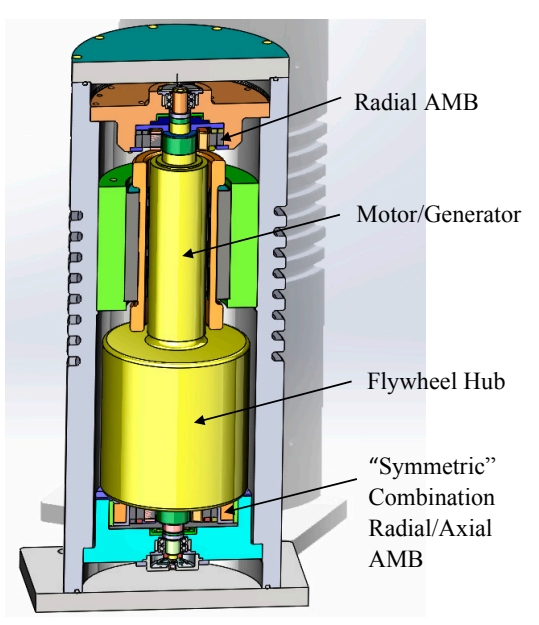

Figure 22. Flywheel energy storage system with homopolar PM-biased AMBs. 


\subsection{Turbocompressors and Gas Blowers}

Over the years, Calnetix has developed a wide range of turbocompressor and gas blowers on magnetic bearings with rotating speeds up to 90,000 RPM and power levels ranging from $60 \mathrm{~kW}$ to 1.2 MW. A typical structure of a single-stage turbocompressor (blower) is shown in Figure 23.

Using either version of the homopolar PM-biased combination actuator ("symmetric" or "side-by-side") in this application would lead to a shorter rotor length, much smaller aerodynamic loss and better performance at speed compared to the conventional arrangement of a dedicated electrically-biased axial actuator per Figure 10 and a radial heteropolar actuator per Figure 7. See [5,6] for a detailed comparison of the designs using different actuator choices. However, the "side-by-side" version has the upper hand here because of two critical advantages: higher frequency of the first bending mode due to the shaft diameter gradually decreasing towards the impeller (even though it would be a little longer axially than with the "symmetric" design) and much better axial bandwidth. The latter is especially important in this application because turbocompressors are known to occasionally experience aerodynamic surge, causing the axial load to become very dynamic.

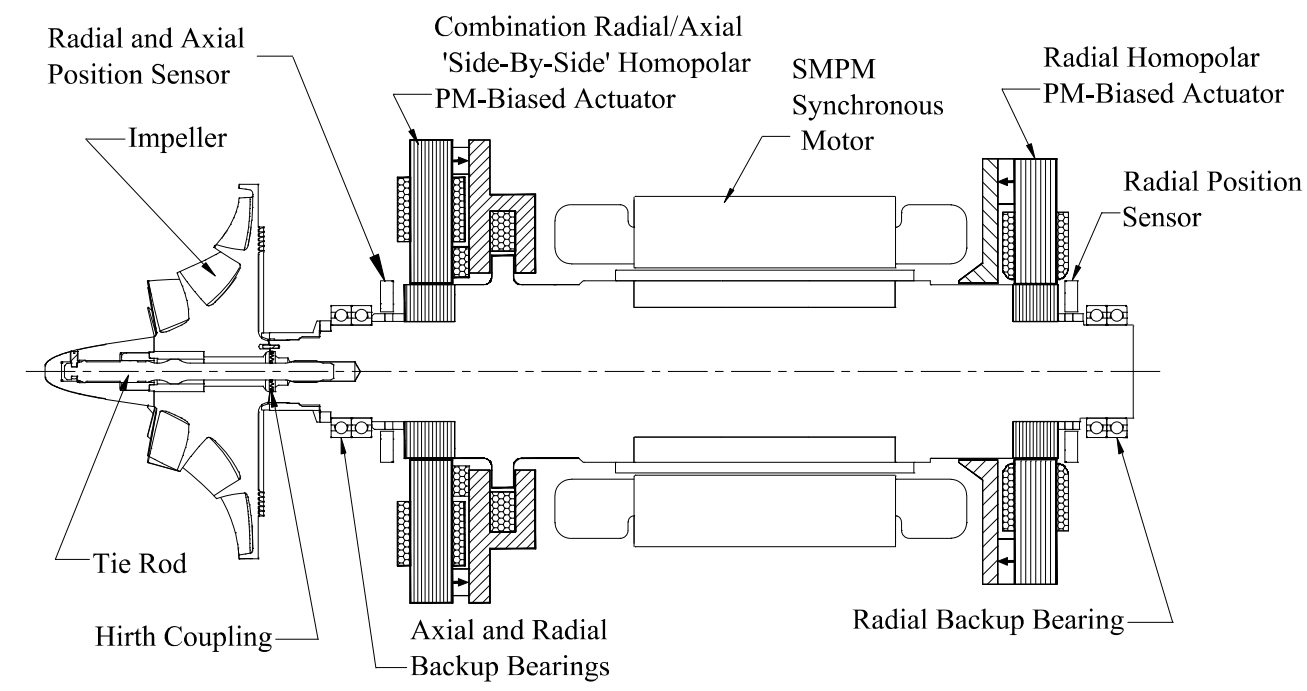

Figure 23. A typical structure of a single-stage turbocompressor (blower) with homopolar PM-biased AMBs.

\subsection{Gas Expanders}

While gas expanders can have a structure fully identical to a typical turbocompressor structure shown in Figure 23, an alternative design with the inverse impeller orientation shown in Figure 24 is used by Calnetix in the expanders developed for its line of the Organic Ranking Cycle machines designed to recuperate electrical energy from various heat sources [16].

Because the impeller (expansion turbine) is located inboard from the combination bearing in this design, the "side-by-side" tapered shaft feature does not lead to a rotordynamic advantage. (The "symmetric" actuator design may even have a slight rotordynamic advantage here because of a shorter axial length; however, we have not verified it). In addition, contrary to the compressors, the expanders do not experience an aerodynamic surge, and therefore, having high axial bandwidth is less important in this application. Even though both "symmetric" and "side-by-side" versions of the combination actuators could be used here, mainly for historic reason the "symmetric" version is used. 


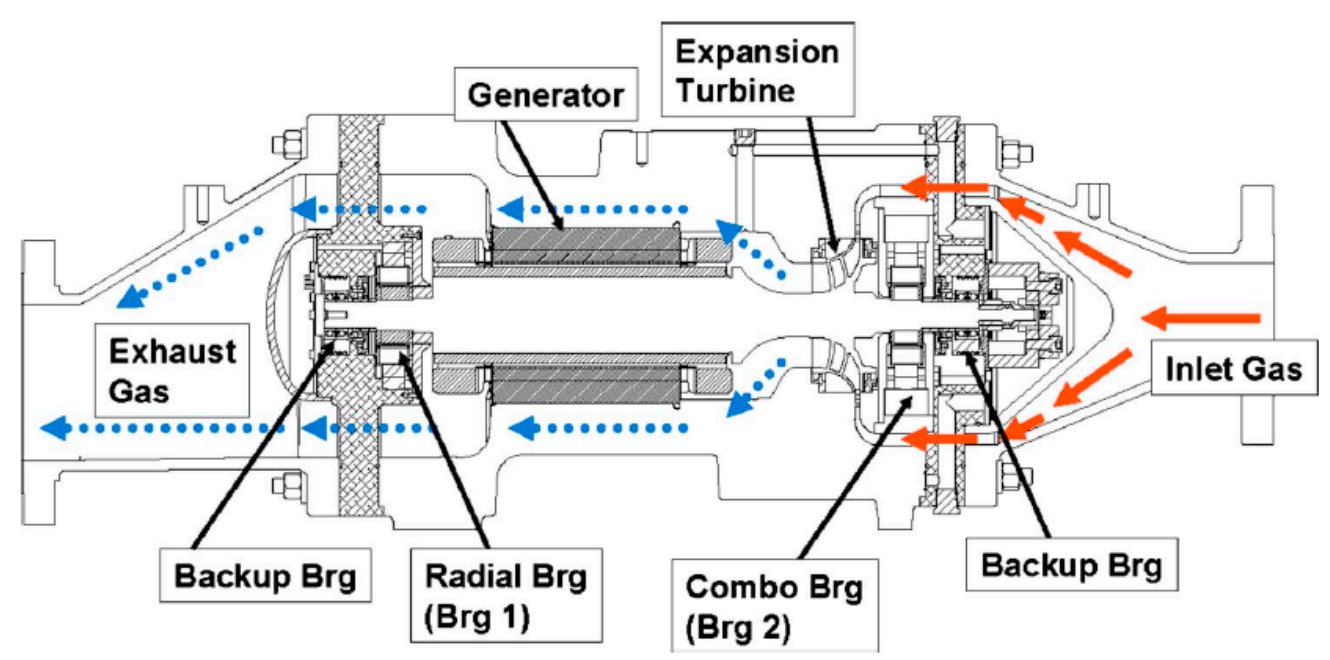

Figure 24. Expander for Organic Ranking Cycle (ORC) heat recovery system with homopolar PM-biased AMBs.

\subsection{Rotary Atomizers}

Rotary atomizers are often used in spray-drying - the preferred method by which the pharmaceutical-processing and food-processing industries produce various dry powders and particles. Figure 25 shows a process flow diagram for a spray-dryer. In a typical spray-drying application, a slurry or solution liquid is fed into a nozzle, which breaks up the fluid into a spray. The nozzle, often a rotary-disc atomizer, creates equal-size droplets. The size of the droplets is inversely proportional to the nozzle/disc peripheral speed; a larger diameter and a higher speed typically yield smaller particle sizes.

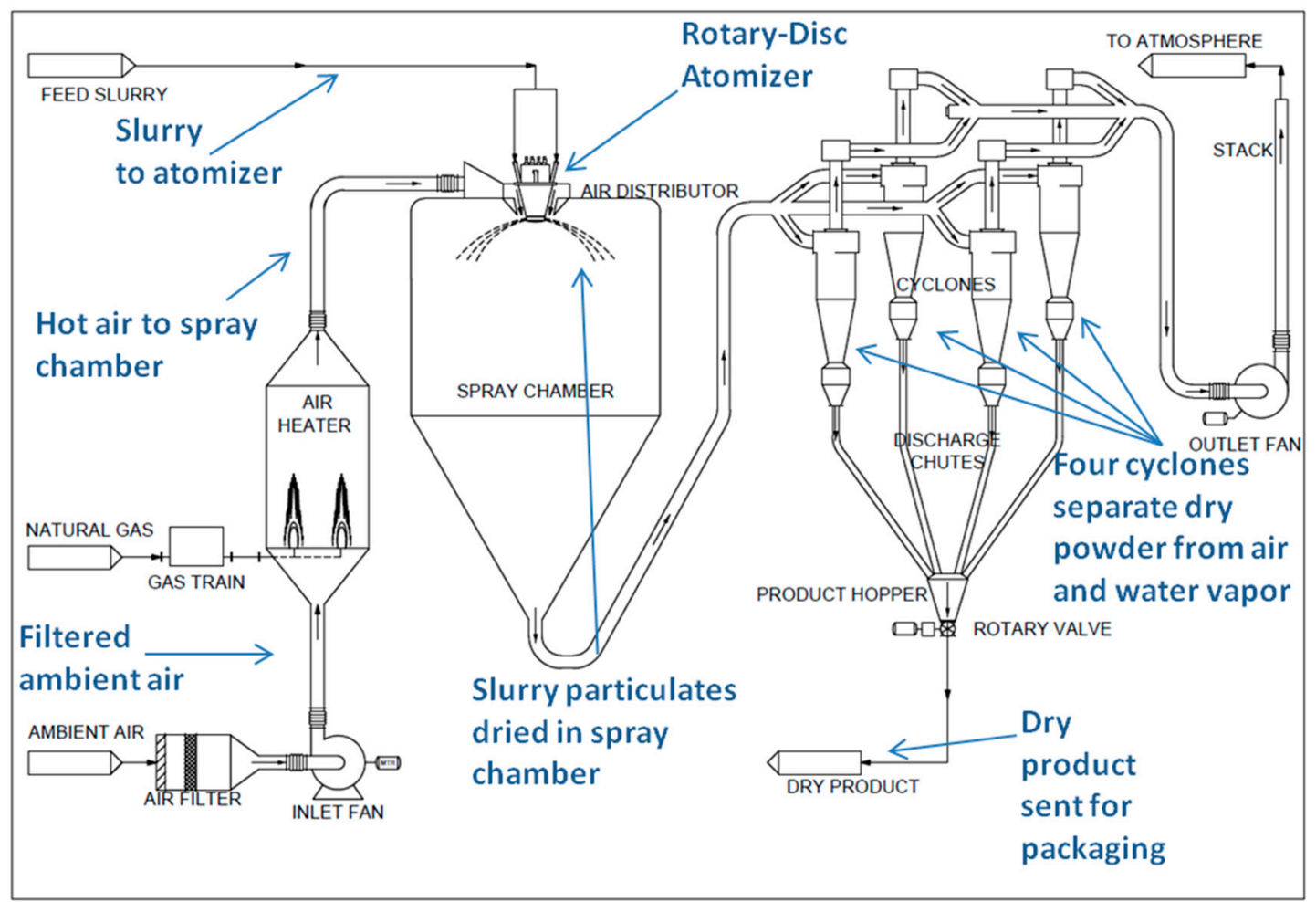

Figure 25. Process flow diagram for a spray-dryer. 
A hot stream of gas, usually air or nitrogen, rapidly dries the droplets to produce equal-size dry powders and particles. The particles produced in modern spray-drying applications typically have a diameter ranging from 0.1 to $0.2 \mathrm{~mm}$.

The cross-section of a rotary atomizer developed at Calnetix is shown in Figure 26. It utilizes a radial homopolar PM-biased AMB at the bottom and a "side-by-side" homopolar PM-biased combination $\mathrm{AMB}$ at the top with a permanent magnet motor in the middle. The machine has a maximum continuous operating speed of 16,000 RPM. More details about this application can be found in [17].

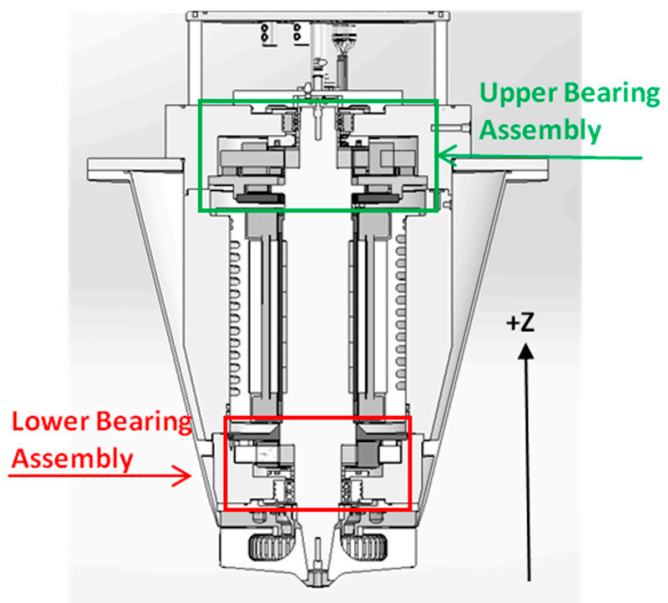

Figure 26. Rotary Atomizer for Spray-Drying with Homopolar PM-Biased AMBs.

\section{Conclusions}

An electromagnetic actuator is the largest part of the AMB system inside a machine, and its design has a big impact on the overall machine performance parameters, including rotordynamic response, ability to react to static and dynamic external forces (particularly at maximal operational speed), both windage and electrical rotational losses, overall machine dimensions, etc. Permanent magnet-biased homopolar actuators are significantly more difficult to design and typically are more expensive than conventional arrangements of electrically-biased heteropolar radial actuators and electrically-biased axial actuators. However, as our 15 years of experience with these actuators show, the payoffs in the machine performance improvements in most cases are well worth the effort.

Conflicts of Interest: The authors declare no conflict of interest.

\section{References}

1. Schweitzer, G.; Maslen, E. Magnetic Bearings: Theory, Design and Application to Rotating Machinery; Springer: Berlin/Heidelberg, Germany, 2009.

2. Stoll, R. The Analysis of Eddy Currents; Oxford University Press: London, UK, 1974.

3. Genta, G. Dynamics of Rotating Systems; Springer: New York, NY, USA, 2005.

4. Filatov, A.V.; McMullen, P.T.; Hawkins, L.A.; Blumber, E.J. Magnetic Bearing Actuator Design for a Gas Expander Generator. In Proceedings of the 9th International Symposium on Magnetic Bearings, Lexington, KY, USA, 3-6 August 2004.

5. Filatov, A.; Hawkins, L. Comparative Study of Axial/Radial Magnetic Bearing Arrangements for Turbocompressor Applications. Proc. Inst. Mech. Eng. I J. Syst. Control Eng. 2016, 230-234, 300-310. [CrossRef]

6. Filatov, A.; Hawkins, L.A. Comparative Study of Axial/Radial Magnetic Bearing Arrangements for Turbocompressor Applications. In Proceedings of the 14th International Symposium on Magnetic Bearings, Linz, Austria, 11-14 August 2014. 
7. McMullen, P.T.; Huynh, C.S.; Hayes, R.J. Combination Radial-Axial Magnetic Bearing. In Proceedings of the 7th International Symposium on Magnetic Bearings, Zurich, Switzerland, 23-25 August 2000.

8. Sortore, C.K.; Allaire, P.E.; Maslen, E.H.; Humphris, R.R. Design of Permanent Magnet Biased Magnetic Bearings for a Flexible Rotor. In Proceedings of the ROMAC Conference, Charlottesville, VA, USA, 2-5 April 1990.

9. Filatov, A.; Hawkins, L.A. Novel Combination Radial/Axial Homopolar Active Magnetic Bearing. In Proceedings of the 1st Brazilian Workshop on Magnetic Bearings, Rio-De-Janeiro, Brazil, 25-26 October 2013.

10. Filatov, A.; Hawkins, L.A.; Ukidve, C. Bias Flux Compensation in the "Side-By-Side" Combination Radial/Axial Homopolar PM-Biased Active Magnetic Bearing. In Proceedings of the 15th International Symposium on Magnetic Bearings, Kitakyushu, Japan, 3-6 August 2016.

11. Filatov, A.; Hawkins, L.A. Combination Axial and Radial Active Magnetic Bearing with Improved Axial Bandwidth. In Proceedings of the ASME Turbo Expo 2012: Power for Land, Sea and Air, Copenhagen, Denmark, 11-15 June 2012.

12. Filatov, A.; Hawkins, L.A. Combination Radial/Axial Electromagnetic Actuator with an Improved Axial Bandwidth. U.S. Patent 8,847,451 B2, 30 September 2014.

13. McCaig, M. Permanent Magnets in Theory and Practice; Pentech Press: London/Plymouth, UK, 1977.

14. Finite Element Method Magnetics. Available online: http://www.femm.info/wiki/HomePage (accessed on 3 November 2016).

15. McMullen, P.; Vuong, V.; Hawkins, L. Flywheel Energy Storage System with AMB's and Hybrid Backup Bearings. In Proceedings of the 10th International Symposium on Magnetic Bearings, Martigny, Switzerland, 21-23 August 2006.

16. Hawkins, L.A.; Zhu, L.; Blumber, E.J. Development of a 125 kW AMB Expander/Generator for Waste Heat Recovery. J. Eng. Gas Turbines Power 2011, 133, 187-194. [CrossRef]

17. Khatri, R.K.; Hawkins, L.A.; Bazergui, C. Demonstrated Operability and Reliability Improvements for a Prototype High-Speed Rotary-Disc Atomizer Supported on Active Magnetic Bearings. In Proceedings of the ASME Turbo Expo 2015: Turbine Technical Conference and Exposition, Montreal, QC, Canada, 15-19 June 2015.

(C) 2016 by the authors; licensee MDPI, Basel, Switzerland. This article is an open access article distributed under the terms and conditions of the Creative Commons Attribution (CC-BY) license (http://creativecommons.org/licenses/by/4.0/). 